Doug Geisler, Eva K. Grebel, and Dante Minniti, eds.

\title{
Ages and Metallicities of Five Intermediate-Age Star Clusters Projected Towards the Small Magellanic Cloud
}

\author{
Juan J. Clariá \& Andrés E. Piatti \\ Observatorio Astronómico, UNC, Laprida 854, 5000 Córdoba, Argentina
}

João F. C. Santos Jr.

Departamento de Física, UFMG, CP 702, 30123-970, Belo Horizonte, Brazil

Eduardo Bica

Instituto de Física, UFRGS, Av. B. Gonçalves 9500, CP 15051, CEP 91501-970, Porto Alegre RS, Brazil

\author{
Ata Sarajedini \\ Department of Astronomy, University of Florida, P.O. Box 112055, \\ Gainesville, Fl 32611 USA \\ Doug Geisler \\ Universidad de Concepción, Departamento de Física, Casilla 160-C, \\ Concepción, Chile
}

\begin{abstract}
Washington CCD photometry of intermediate-age clusters in the Small Magellanic Cloud (SMC) is presented. The cluster age distribution in the SMC suggests formation epochs at 3 and 6 Gyr, respectively. Recent star formation is confined to the central body of the SMC. The chemical evolution of the SMC appears to be best represented by bursty models.
\end{abstract}

\section{Cluster sample}

We present for the first time colour-magnitude diagrams (CMDs) in the Washington system for five star clusters (L 32, L 38, K 28, K 44 and L 116) and their surrounding fields, which lie in or are projected onto the SMC. The observations were performed with the CTIO $0.9 \mathrm{~m}$ telescope in 1998 . After applying a careful cleaning procedure of these CMDs, we determined age and metallicity for both the clusters and their respective surrounding fields, following the methods described by Geisler et al (1997) and Geisler \& Sarajedini (1999).

\section{Main results}

Briefly, we draw the following main conclusions: 
1) L 116 probably does not belong to the SMC but to the LMC, according to the distance estimate based on the red giant clump magnitude. The cluster is closer to the LMC than to the SMC.

2) All the clusters turned out to be of intermediate-age, with ages ranging from 2 to $6 \mathrm{Gyr}$, which increases the number of intermediate-age and old clusters presently known in the SMC with ages and metallicities determined on a uniform scale to 11 .

3) Using our data and those of previous studies, we investigated the cluster age distribution in the SMC. The frequency distribution of clusters with age suggests two important cluster formation epochs in the SMC: one at 3 Gyr and another at 6 Gyr, as can be seen in Figure 1, although more observations are needed for a better definition of these two events.

4) Concerning the SMC field population, we found that in the inner fields a young stellar population is mixed with the intermediate-age one, while in the outer fields the intermediate-age component is dominant and the young component does not show up. This demonstrates that recent star formation has occurred preferentially in regions close to the SMC main body.

5) We studied the chemical enrichment of the SMC using our data and those of Mighell et al. (1998) and Da Costa \& Hatzidimitriou (1998). We show in Figure 2 that a closed box continuous star formation model (dashed line) is a poor representation of the SMC star formation history. Instead, the refinement of the bursting model (solid line), such as proposed by Pagel \& Tautvaisiene (1998), is closer to the observed data points. More details can be found in Piatti et al. (2001).

\section{References}

Geisler, D., Bica, E., Clariá, J.J, Piatti, A.E. \& Santos Jr., J.F.C. 1997, AJ, 114,920

Geisler, D. \& Sarajedini, A. 1999, AJ, 117, 308

Da Costa, G.S. \& Hatzidimitriou, D. 1998, AJ, 115, 1934

Mighell, K.J., Sarajedini, A. \& French, R.S. 1998, AJ, 116, 2414

Pagel, B.E.J. \& Tautvaisiene, G. 1998, MNRAS, 299, 535

Piatti, A.E., Santos Jr., J.F.C., Clariá, J.J., Bica, E., Sarajedini, A. \& Geisler, D. 2001, MNRAS, 325, 792 


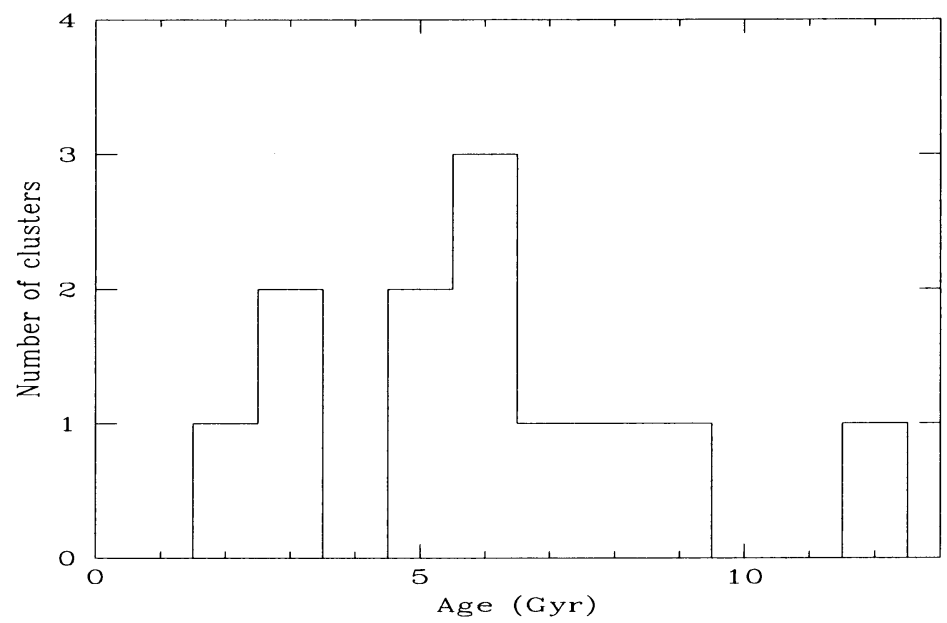

Figure 1. The age distribution of SMC clusters older than $1 \mathrm{Gyr}$ derived by Mighell et al. (1998) and the present study.

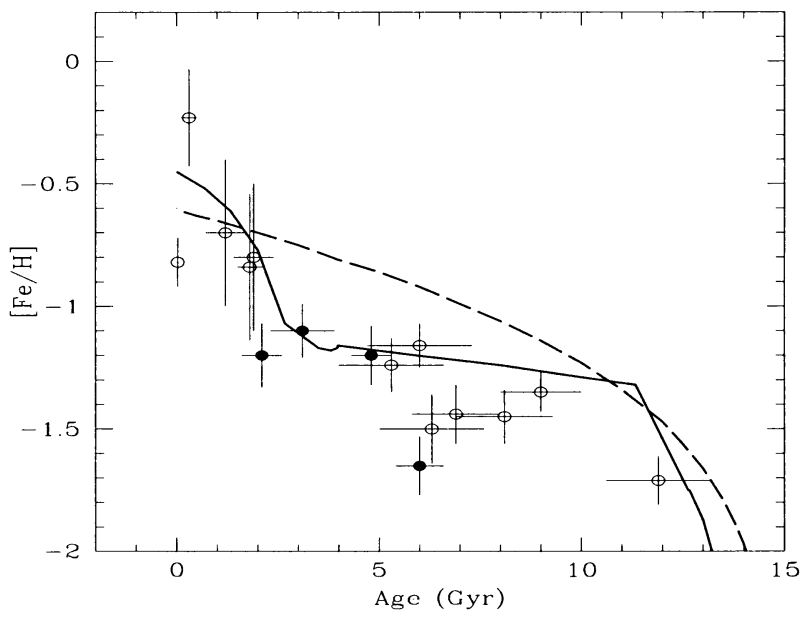

Figure 2. Age-metallicity relationship for star clusters in the SMC. Open circles represent data previously published by Da Costa \& Hatzidimitriou (1998) and Mighell et al. (1998), while filled circles correspond to the SMC clusters studied in this work. Error bars are also included. The data are compared with the closed box continuous star formation model (dashed line) computed by Da Costa \& Hatzidimitriou (1998) for an assumed present day SMC metallicity of -0.6 dex, and the bursting model (solid line) of Pagel \& Tautvaisiene (1998) 